\title{
Gesundheitsförderung: ein Schlüsselbeitrag zur nachhaltigen Entwicklung
}

\author{
Die 12. Nationale Gesundheitsförderungs-Konferenz findet am 6. und 7. Januar 2011 \\ in Davos statt. Thematisiert werden vor allem die Healthy3-Initiative und der Zusam- \\ menhang zwischen Gesundheitsförderung und nachhaltiger Entwicklung.
}

Catherine Favre Kruit

Gesundheitsförderung Schweiz
Korrespondenz: Catherine Favre Kruit Projektleiterin Partner Relations Promotion Santé Suisse Avenue de la Gare 52 CH-1003 Lausanne Tel. 0213451515 Fax 0213451545

catherine.favre@ promotionsante.ch

www.gesundheitsfoerderung.ch
Zwischen Gesundheitsförderung und nachhaltiger Entwicklung gibt es viele Parallelen. Gemeinsamkeiten finden sich sowohl in den Grundsätzen wie auch in der Zielsetzung. Die sozialen, wirtschaftlichen und umweltbezogenen Komponenten, die auf die Gesundheit einwirken, sind auch die entscheidenden Determinanten für eine nachhaltige Entwicklung. Und schliesslich muss Gesundheit auch als eines der wichtigsten Anliegen nachhaltiger Entwicklung gelten!

\section{Die Thematik der Konferenz}

Die 12. Nationale Gesundheitsförderungs-Konferenz vom 6. und 7. Januar 2011 wird von «Gesundheitsförderung Schweiz» gemeinsam mit dem Kanton Graubünden organisiert und durchgeführt. Dieser Anlass wird eine erste Gelegenheit bieten, die Denkanstösse der Weltkonferenz über die Verknüpfung von Gesundheitsförderung und nachhaltiger Entwicklung vom Juli 2010 in Genf aufzunehmen und auf nationaler Ebene weiterzuführen. Eine zentrale Frage beider Konferenzen wird sein: Welchen Beitrag kann die Gesundheitsförderung an eine nachhaltige Entwicklung leisten? Und umgekehrt: Was ist der Beitrag der nachhaltigen Entwicklung an die Gesundheitsförderung? Es gilt, in den vielfältigen Bezügen zwischen Theorie und Praxis (beziehungsweise zwischen Policy und Praxis) Gemeinsamkeiten zu entdecken und dabei im gegenseitigen Austausch voneinander zu lernen.

Im Zentrum des Konferenzprogramms stehen die Plenar- und Subplenarveranstaltungen, welche die Möglichkeit bieten sollen, eine Bilanz über die Weltkonferenz (www.iuhpeconference.net) zu ziehen und die Verbindungen zwischen Gesundheitsförderung und nachhaltiger Entwicklung zu vertiefen. Ein spezielles Augenmerk wird dabei auf die Themen Partizipation, Verhaltensänderungen und sektorübergreifendes Arbeiten gerichtet, welche sowohl für die Gesundheitsförderung wie auch für die nachhaltige Entwicklung von grosser Bedeutung sind. Für jedes der oben erwähnten Themen werden verschiedene Workshops die Möglichkeit bieten, in Kleingruppen praktische Erfahrungen auszutauschen.

\section{Healthy3-Initiative}

Der zweite Konferenztag ist unter anderem der Vorstellung der «Healthy3-Initiative» gewidmet, die von

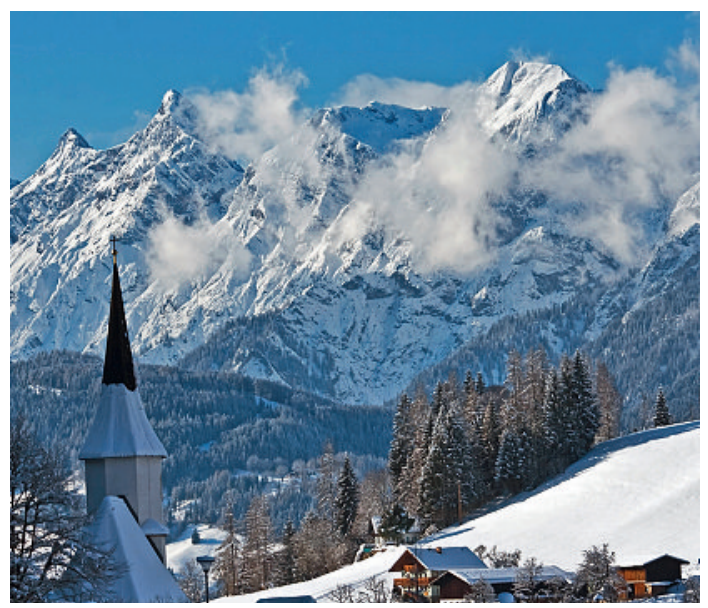

Die Gesundheitskonferenz will hoch hinaus auf 1540 Meter über dem Meer.

«Gesundheitsförderung Schweiz» an der Weltkonferenz in Genf lanciert wurde. Die Initiative verfolgt das Ziel, eine Brücke zwischen der Gesundheitsförderung und der nachhaltigen Entwicklung zu schlagen. Als erstes Handlungsfeld stehen die Themen «Nahrung» und «Ernährung» im Mittelpunkt der Initiative, mit welchen die heutigen und zukünftigen Herausforderungen sowohl der Gesundheitsförderung wie auch der nachhaltigen Entwicklung konfrontiert sind. An einer Podiumsdiskussion mit Vertretern des Gesundheitssektors, der Politik und der Wirtschaft werden schliesslich Wege für mögliche Umsetzungen in der Schweiz vorgestellt und diskutiert.

Die Veranstalter wünschen sich für die Konferenz eine aktive Teilnahme von Behördenvertreterinnen und -vertretern, von Fachpersonen aus ärztlichen Kreisen, wissenschaftlichen Bereichen, Politikerinnen und Politikern sowie von Akteuren und Angehörigen der Berufe im Bereich von Gesundheit und nachhaltiger Entwicklung. So können die erforderlichen Allianzen und Partnerschaften entstehen, die in der Politik und im praktischen Alltag nötig sind, um die Gesundheit der Bevölkerung positiv zu beeinflussen.

Programm der Konferenz und Anmeldung: www.gesundheitsfoerderung.ch/konferenz 